\title{
REPERCUSSÕES DA SÍNDROME PRÉ- MENSTRUAL EM ESTUDANTES \\ UNIVERSITÁRIAS: UMA REVISÃO
}

CATEGORIA: CLÍNICO

CENTRO UNIVERSITÁRIO SÃo CAMILO

Giannetto, $B^{1}$ - Endereço Av. Hilário Pereira de Souza, número 492 - Telefone: (11) 98224 1038 - Email: beagiannetto@gmail.com

Tamajusuku, E ${ }^{1}$

Gastaldo, LPC $^{1}$

Vellenich, GAH $^{1}$

Pereira, $\mathbf{M M}^{2}$

1 Discente do Centro Universitário São Camilo

2 Docente do Centro Universitário São Camilo 
REPERCUSSÕES DA SÍNDROME PRÉ-MENSTRUAL EM ESTUDANTES UNIVERSITÁRIAS: UMA REVISÃO

CATEGORIA: CLÍNICO

DESCRITORES: "Síndrome Pré-Menstrual”, "Estudantes" 


\section{RESUMO}

INTRODUÇÃO: Os sinais e sintomas causados pela Síndrome Pré-Menstrual (SPM) acometem cerca de 75 a $80 \%$ da população. As consequências da Síndrome Pré-Menstrual apresentadas pelas mulheres interfere de forma significativa na vida destas atrapalhando as atividades diárias e, consequentemente, o desempenho no meio acadêmico.

METODOLOGIA: Realizada revisão bibliográfica na base de dados Pubmed, no período entre 2015-2020, utilizando-se os descritores "Premenstrual Syndrome" and "Female students". Após aplicar os filtros: língua portuguesa, inglesa e espanhola, excluindo artigos de revisão e relatos de caso, foram selecionados 14 artigos. OBJETIVO: Avaliar as repercussões da Síndrome Pré-Menstrual em estudantes universitárias.

RESULTADOS: A média de apresentação da SPM foi de 59\%, sendo que os principais sintomas foram depressão $(83,9 \%)$, dor $(83,9 \%)$ e outros sintomas físicos $(64,3 \%)$, estresse $(77,8 \%)$, insônia ou hipersonia $(54,6 \%)$, fadiga $(59 \%)$ e dismenorreia $(41,9 \%)$. Os impactos na vida acadêmica foram: prejuízos de autodeterminação e motivação acadêmica, produtividade (48,3\%), atividades de vida social (19\%), relacionamentos $(28,7 \%)$ e na performance em geral $(44,3 \%)$. Os principais fatores relacionados à piora dos sintomas da SPM foram a ingestão de alimentos com alto teor calórico, de gordura, açúcar e sal, IMC, etilismo, tabagismo e história familiar. Os sintomas físicos aumentam conforme há ingestão de alimentos com alto teor calórico, de gordura, açúcar e sal (OR 3,2, IC 95\% 1,4- 7,3; $\mathrm{p}<0,05)$; o consumo de frutas foi associado a diminuição dos sintomas comportamentais (OR 0,34, IC 95\% 0,125-0,92; $\mathrm{p}<0,05$ ); níveis mais altos de vitamina D diminuiam sintomas da SPM; o tabagismo e o etilismo foram associado ao aumento do risco de relatar sintomas psicológicos (OR 2,5, IC 95\% 1,1-5,8; $\mathrm{p}<0,05)$. Somado a isso, mulheres que apresentavam anemia, doenças crônicas, sedentarismo e história familiar de Síndrome Pré-Menstrual tinham sintomas mais intensos.

CONCLUSÃO: A produtividade, determinação e, sobretudo o desenvolvimento acadêmico das estudantes podem ser afetados devido aos sinais e sintomas que as mulheres com Síndrome PréMenstrual apresentam. Há um agravo nos sinais e sintomas de acordo com o estilo de vida que essas mulheres levam.

DESCRITORES: "Premenstrual Syndrome", "Female students" 


\begin{abstract}
INTRODUCTION: The signs and symptoms caused by Premenstrual Syndrome (PMS) affects about 75 to $80 \%$ of the population. The consequences of PMS presented by women significantly interfere with their lives, disrupting daily activities and, consequently, performance in the academic environment.

METHODOLOGY: A (systematic or bibliographic) review was made using the PubMed database, using the time period of 2015-2020 and the descriptors "Premenstrual Syndrome and Female students". After using the filters of articles in Portuguese, Spanish and English and excluding review articles and case reports, 14 original articles were used. OBJECTIVE: Evaluate the repercussion of the premenstrual syndrome in female students.

RESULTS: The average presentation of PMS was 59\%, with the main symptoms being depression $(83,9 \%)$, pain $(83,9 \%)$ and other physical symptoms $(64,3 \%)$, stress $(77,8 \%)$, insomnia or hypersomnia $(54,6 \%)$, fatigue $(59 \%)$ and dysmenorrhea $(41,9 \%)$. The impacts on academic life were: impairment of self-determination and academic motivation, productivity $(48,3 \%)$, social life activities (19\%), relationships $(28,7 \%)$ and performance in general $(44,3 \%)$. The main factors related to the worsening of PMS symptoms were the ingestion of foods with high caloric content, fat, sugar and salt, BMI, alcohol consumption, tabagism and family history. Physical symptoms increases according to the intake of foods with a high caloric content, fat, sugar and salt (OR 3,2, IC 95\% 1,4- 7,3; $p$ $<0,05$ ); fruit consumption was associated with a decrease in behavioral symptoms (OR 0,34, IC 95\% $0,125-0,92 ; \mathrm{p}<0,05)$; higher levels of vitamin D decreased symptoms of PMS; smoking and drinking were associated with an increased risk of reporting psychological symptoms (OR 2,5, IC 95\% 1,1$5,8 ; \mathrm{p}<0,05)$. In addition, women with anemia, chronic diseases, physical inactivity and family history of PMS had more severe symptoms.

CONCLUSION: The productivity, determination and, above all, the academic development can be affected according to the signs and symptoms that women with PMS presents. There is an aggravation of signs and symptoms in accordance with the lifestyle these women have.
\end{abstract}

Keywords: Premenstrual syndrome and Female students 


\section{REPERCUSSÕES DA SÍNDROME PRÉ- MENSTRUAL EM ESTUDANTES UNIVERSITÁRIAS: UMA REVISÃO}

\section{INTRODUÇÃO}

Durante o período reprodutivo, a síndrome pré-menstrual (SPM) atinge, de forma recorrente cerca de 75 a $80 \%$ das mulheres interferindo em inúmeros âmbitos da sua vida. A SPM é um conjunto de sinais e sintomas físicos, emocionais e comportamentais. A tensão pré-menstrual (TPM) como também é conhecida a SPM apresenta caráter cíclico, iniciando-se durante a fase lútea e que é aliviada com a menstruação'.

O mecanismo etiológico da SPM ainda não foi totalmente esclarecido, entretanto os neurotransmissores - serotonina e ácido gama-aminobutírico (GABA) sob efeito do estradiol e progesterona e a atividade cíclica dos ovários são uma possível explicação acerca da fisiopatologia da síndrome. Outras etiologias possivelmente envolvidas são as endorfinas, fatores da homeostase do cálcio, causas ambientais e deficiência de vitamina B6 e magnésio ${ }^{1}$. Além disso, a etiologia pode estar relacionada a aspectos sociais, ambientais, psicológicos, hormonais e familiares ${ }^{2}$.

A presença de um ou mais sintomas somáticos e/ ou emocionais durante os cinco dias que antecedem o ciclo menstrual ou durante a fase lútea compõe o diagnóstico da síndrome, de acordo com o American College of Obstetricians and Gynecologists (ACOG). Quando se encontram até três sintomas a SPM é considerada leve, e até 4 sintomas é considerada moderada ${ }^{1}$.

Geralmente, são observados os seguintes sinais e sintomas: edema de abdômen e extremidades, ganho de peso, aumento do tamanho e maior sensibilidade nas mamas, cefaleia, fadiga, acne, ansiedade, irritabilidade, mudanças de humor, entre outros.

No meio acadêmico, esses sintomas são fomentados pela árdua rotina das estudantes que levam um estresse cumulativo interferindo demasiadamente na qualidade de vida e desempenho nas atividades diárias das mulheres. Tal estresse é resultado tanto do estresse psicológico quanto do estresse físico, que ocorre durante o ciclo menstrual ${ }^{1}$.

Somado às situações adversas dessa síndrome, nota-se que o estilo de vida levado por essas estudantes, como a alimentação, exercícios físicos, ingestão de bebidas alcoólicas e tabagismo, por exemplo, muitas vezes, contribui para os sintomas da SPM. Com a piora dos sintomas da SPM, há prejuízo em diversos âmbitos da vida da mulher, como queda da produtividade, dificuldade em atividades na vida social e nos relacionamentos ${ }^{3}$.

Dessa forma, o objetivo desta revisão é avaliar as repercussões da síndrome pré-menstrual em estudantes universitárias.

PALAVRAS-CHAVE: Premenstrual Syndrome and Female students

\section{METODOLOGIA}

Foi realizada uma revisão bibliográfica na base de dados PubMed utilizando os descritores "Premenstrual Syndrome and Female students"; no período de 2015 a 2020. Após essa busca, foram encontrados 75 artigos. Foram incluídos estudos que respondiam à questão norteadora desta revisão e após aplicação dos critérios de exclusão: artigos que não estavam nas línguas portuguesa, inglesa e espanhola, artigos de revisão e relatos de caso, foram utilizados 14 estudos originais.

\section{RESULTADOS}

Os resultados encontrados com a realização da revisão bibliográfica estão na tabela abaixo: 


\begin{tabular}{|c|c|c|c|c|}
\hline Título do artigo & $\begin{array}{l}\text { Ano de } \\
\text { publicação e } \\
\text { tipo de artigo }\end{array}$ & Amostra & Resultados & Conclusão \\
\hline $\begin{array}{l}\text { Prevalence of } \\
\text { premenstrual } \\
\text { syndrome and } \\
\text { its impact on } \\
\text { quality of life } \\
\text { among selected } \\
\text { college students } \\
\text { in Puducherry }\end{array}$ & $\begin{array}{l}2019 \text { - Estudo } \\
\text { transversal }\end{array}$ & $\begin{array}{l}300 \text { estudantes } \\
\text { do curso de } \\
\text { ciências da } \\
\text { faculdade } \\
\text { feminina de } \\
\text { Puducherry }\end{array}$ & $\begin{array}{l}\text { A prevalência de } \\
\text { SPM foi de } 62,7 \% \\
\text { entre as estudantes de } \\
\text { uma faculdade que } \\
\text { frequentavam cursos } \\
\text { de ciências. Os } \\
\text { sintomas da SPM } \\
\text { mais comuns foram } \\
\text { dores no corpo, } \\
\text { mialgia e artralgia } \\
(71,3 \%), \text { peso e } \\
\text { desconforto (64,3\%). } \\
\text { abdominal } \\
\text { O estilo de vida como } \\
\text { atividade física, } \\
\text { consumo de cafeína, } \\
\text { doces e junk food } \\
\text { tiveram uma } \\
\text { associação com a } \\
\text { SPM. }\end{array}$ & $\begin{array}{lr}\text { As participantes } \\
\text { com } & \text { SPM } \\
\text { tiveram } & \text { pior } \\
\text { qualidade } & \text { de } \\
\text { vida do } & \text { que } \\
\text { aquelas } & \text { sem } \\
\text { SPM. } & \end{array}$ \\
\hline
\end{tabular}

Negative Mood 2019 - Estudo 88 estudantes O humor negativo Ingerir chá, café Is Associated transversal do sexo associou-se à ingestão e de bebidas with Diet and feminino de chá, café e bebidas carbonatadas e a Dietary

Antioxidants in University

Students During the Menstrual Cycle: A CrossSectional Study

from

Guangzhou, China (primeiro, no segundo e terceiro ano ou pós-graduados de todas as universidades da cidade de) Guangzhou, China carbonatadas durante frequência de a fase menstrual $(\mathrm{p}=$ consumo dessas $0,0453, \mathrm{OR}=1,23)$ e foi mais alta no o humor negativo grupo com associou-se maior positivamente à pontuação para ingestão de banana humor negativo durante o período pré- do que no grupo menstrual $(\mathrm{p}=\mathrm{com}$ baixa $0,0172, \mathrm{OR}=1,81) \quad$ pontuação durante a fase menstrual. As universitárias com um humor mais negativo têm maior chance dessas bebidas para estimular $\quad 0$ sistema nervoso e aliviar o humor negativo. 


$\begin{aligned} & \text { Comparison of } \\ & \text { serum level of } \\ & \text { trace }\end{aligned}$
some $\quad$ transversal
elements and
vitamin
between
patients with
premenstrual
syndrome and
normal
controls: A
cross-sectional
study.

Premenstrual 2019 - Estudo 285 estudantes Syndrome in transversal de um hospital Students of a Teaching Hospital.
300 estudantes do sexo feminino (19 a 21 anos) do condado de Falavarjan de ensino.
Quality of Life 2019 - Estudo 642 estudantes among University

Students with

Premenstrual Syndrome.
A prevalência de SPM foi de cerca de 41,5\%. O nível de vitamina $\mathrm{D}$ diminuiu nos grupos controle e SPM, com uma faixa menor de vitamina $\mathrm{D}$ $(p \leq 0,05)$ no grupo SPM. $O$ maior percentual de de Portanto, para sintomas psicóticos reduzir os nos grupos da SPM sintomas da foi irritabilidade SPM, o nível $(53,91 \%)$, depressão sérico de $(30,43 \%)$ e ansiedade vitamina D deve $(21 \%)$, e o percentual estar no nível máximo de sinais físicos foram sensibilidade da mama $(21,74 \%)$, distensão abdominal $(19,13 \%)$ e cansaço geral (18\%).

\section{6 \\ $(72,3 \%)$}

relataram pelo menos um sintoma de SPM de intensidade moderada a grave, dentre as quais 74 $(25,9 \%)$ apresentaram pelo menos um sintoma grave. 6 $(2,1 \%)$ preencheram todos os critérios para TDPM e $49(17,2 \%)$ preencheram os critérios para SPM moderada a grave e o restante $(80,7 \%)$ apresentava SPM inexistente ou leve, com sintomas isolados.

Das 642 estudantes, SPM leve e 49,9\% tinham SPM, TDPM são $23,3 \%$ apresentavam prevalentes SPM leve e 26,6\% entre estudantes apresentavam TDPM. universitários A maioria das em cursos estudantes tinha entre relacionados à 
18 e 24 anos, tinham ciclos menstruais regulares praticavam atividade física.

diferença

estatisticamente

significante

domínios físico e

mental entre os

estudantes que não

tinham SPM e aqueles

que apresentavam

SPM leve ou SPM ( $p$ $<0,001)$. O consumo de chocolate, cafeína e álcool tem sido relacionado ao TDPM.

Premenstrual Syndrome Is 2019 - Estudo Associated with

Dietary and

Lifestyle

Behaviors among

University

Students: A

Cross-Sectional

Study from

Sharjah, UAE.
300 estudantes universitárias adultas com idades entre 18 e 24 anos
95\% das participantes relataram pelo menos um sintoma da SPM durante $o$ período menstrual. prevalência de SPM foi de $35,3 \%$, sendo mais relatados sintomas leves. $\mathrm{O}$ tabagismo está associado ao aumento do risco de relatar sintomas psicológicos (OR 2,5, IC 95\% 1,1$5,8 ;$ p $<0,05)$ e comportamentais (OR 2,2, IC 95\% 1,04,9; $\mathrm{p}<0,05)$, enquanto a ingestão de alimentos com alto teor calórico, de gordura, de açúcar, de sal foi associada ao aumento do risco de relatar sintomas físicos (OR 3,2, IC 95\% 1,4-7,3; p $<0,05)$. No entanto, o consumo de frutas (OR 0,34, IC 95\% $0,125-0,92 ; p<0,05)$ foi associado a uma saúde, e a SPM

pode afetar a

autoavaliação

das alunas em

todos os

domínios da

qualidade de vida.

(p)

Foi relatada alta prevalência de SPM entre estudantes universitárias, com tabagismo e alto consumo de calorias/ gordura/ açúcar/ sal, identificados como fortes fatores de risco para a SPM. 
diminuição do risco

de relatar sintomas

comportamentais.

Prevalence of 2018 - Estudo 370 mulheres Premenstrual prospectivo na graduação

Syndrome and

Changes in

Blood Pressure

with Menstrual

Cycle Among

University

Students.
2018 - Estudo 448

transversal

Syndrome:

Existence,

Knowledge, and

Attitude Among

Female

University

Students in

Karachi por
Dor nas costas, desejo Alta prevalência de ficar sozinha, de SPM, com artralgia ou mialgia, maioria

fadiga e dor na coxa indicando que foram os 5 sintomas os sintomas são mais relatados. Cerca leves a graves. de $89 \%$ das A pressão participantes arterial relataram aumentou experimentar pelo significativamenos um sintoma mente na fase por ciclo. A pressão lútea do que na arterial média, a fase folicular. pressão arterial sistólica e diastólica foram significativamente $(\mathrm{P}$ $<0,001)$ maiores na fase lútea do que na fase folicular.
A maioria $(96,4 \%)$
Há um impacto das alunas tinha significativo da conhecimento
da SPM na vida SPM, enquanto das mulheres apenas $19 \%$ das paquistanesas e mulheres conheciam é um problema o TDPM. A comum em todo prevalência $\mathrm{O}$ mundo. autorreferida de SPM Embora haja foi de $79,5 \%$ e a uma maior prevalência conscientização utilizando os critérios, ainda existe ACOG foi de $23,9 \%$. uma Os sintomas comuns considerável foram irritabilidade, deficiência de explosões de raiva, conhecimento depressão, sensibilidade mamária e problemas gastrointestinais.
Mais da metade $(60,4 \%) \quad$ dos sobre a necessidade de consultar um médico ou participantes relatou procurar que a SPM perturbou sua rotina normal, enquanto $\quad 81,5 \%$ relataram que 0 tratamento para seus sintomas. universitárias em Karachi selecionados amostragem aleatória entre 18 e 30 anos de idade 
estresse exacerbou seus sintomas. A maioria $(77,5 \%)$ das mulheres acreditava que a SPM era uma questão significativa a ser discutida, mas $49,4 \%$ não fizeram tratamento para a SPM.

\section{Premenstrual life quality in Turkish health science students} syndrome and longitudinal

$\begin{aligned} & \text { Fish } 2016-\text { Estudo } \\ & \text { Consumption } \\ & \text { and }\end{aligned}$
transversal
Premenstrual
Syndrome and
Dysphoric
Disorder in
Japanese
Collegiate
Athletes

608

voluntárias

estudantes da área da saúde em uma universidade estadual na Turquia

\section{2}

estudantes, sendo $\quad 200$ participantes de esportes e 112 não participantes
Não houve diferença A frequência da entre os sintomas da SPM foi de SPM dependendo da $84,5 \%$ e os situação sociodemográfica, mulheres com IMC menor que $18,5 \mathrm{~kg} / \mathrm{m}^{2}$ tinham escores de sintomas maiores, uso de álcool e consumo de fast food aumentavam o escore. irregular. Os $32,7 \%$ tinham sintomas mais sintomas leves, 33,2 comuns foram tinham sintomas fadiga $(100 \%) \mathrm{e}$ moderados e $18,6 \%$ explosões de tinham sintomas raiva $(98,2 \%)$. severos história Dor e humor familiar e deprimido menstruação irregular apareceram em também aumentavam 96,5\% das o escore.

\section{participantes.}

Conforme aumentava a severidade da SPM diminuía a qualidade de vida.

A severidade dos sintomas físicos da SPM e a performance em treinamento e competição foi maior nas participantes que faziam esportes (em mulheres com sintomas severos a porcentagem dos
O estudo tem diversas limitações, mas demonstrou-se que o consumo de peixe tem uma relação com melhora dos sintomas da SPM. Isso se dá por dois mecanismos: 
sintomas físicos em atletas foi de $9 \% \mathrm{e}$ $5,4 \%)$. O consumo de peixe foi associado a diminuição do risco de performance ruim em atletas. nas não atletas foi de

ômega 3 tem ação benéfica para os sintomas da SPM e o consumo de peixe parece ter uma relação positiva com a melhora da performance. $\mathrm{O}$ ômega 3 atua com diminuição da fadiga $\mathrm{e}$ melhora a função neuromotora.
Nature and 2016 - Estudo 285 alunas
Prevalence of observacional

Menstrual

Disorders

among Teenage

Female

Students at

Zagazig

University,

Zagazig, Egypt com menos de

20 anos
$66 \%$ das alunas apresentaram

dismenorreia. $56,1 \%$ das participantes relataram pelo menos um sintoma da SPM. Foi demonstrado que o aumento do IMC tem relação com os sintomas da SPM.

Apesar das limitações desse estudo, esse foi um dos únicos que levou em conta essa população; os dados apresentados não variam significativamente de outros dados ao redor do mundo.

Premenstrual 2015 - Estudo
Syndrome and transversal
Premenstrual
Dysphoric
Disorder in
Japanese
Collegiate
Athletes

174 atletas
universitárias
entre 18 e 23
anos

$53,3 \%$ das Uma intensa participantes carga de apresentaram humor trabalho e deprimido, 73,6\%, estresse severo raiva ou irritabilidade, pioram os $79,5 \%$, ansiedade ou sintomas da tensão, 71,8\%, fadiga SPM/ TDPM. O ou falta de energia, estresse pode $54,6 \%$, insônia ou alterar o sistema hipersonia, $71,3 \%$, neuroendócrino sintomas físicos. Os e induzir os sintomas afetaram a sintomas de produtividade em SPM/ TDPM. $48,3 \%$, as atividades Os treinos e de vida social em competições $19 \%$ os aumentam o relacionamentos em estresse nas $28,7 \%$ e performance atletas. em $44,3 \%$. 


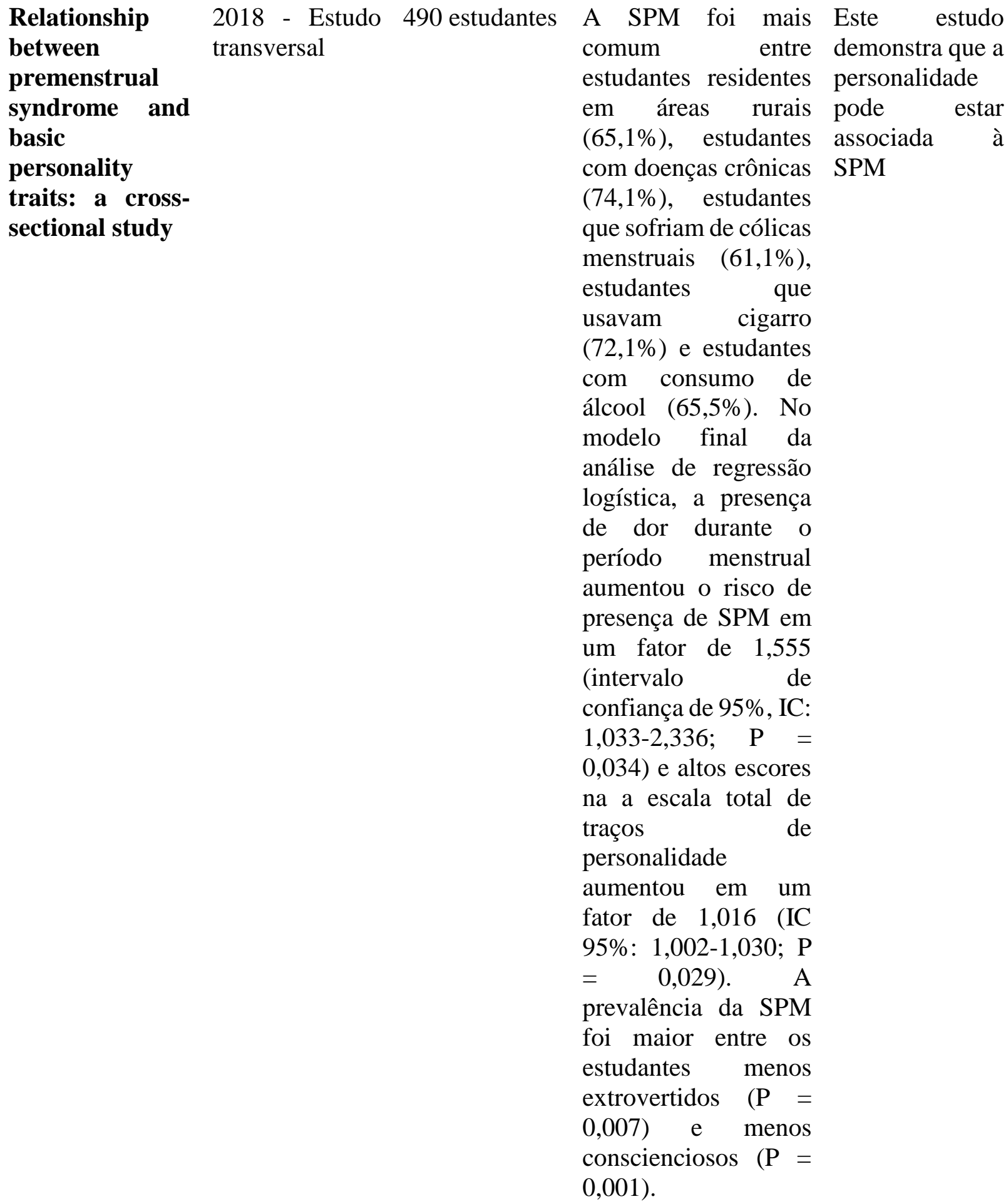




\begin{tabular}{|c|c|c|c|}
\hline $\begin{array}{l}\text { Menstrual } \\
\text { characteristics, } \\
\text { disorders and } \\
\text { associated risk } \\
\text { factors among } \\
\text { female } \\
\text { international } \\
\text { students in } \\
\text { Zhejiang } \\
\text { Province, } \\
\text { China: a cross- } \\
\text { sectional } \\
\text { survey. }\end{array}$ & $\begin{array}{l}2019 \text { - Estudo } \\
\text { transversal }\end{array}$ & $\begin{array}{l}409 \\
\text { questionários } \\
\text { atenderam aos } \\
\text { critérios } \\
\text { foram } \\
\text { utilizados para } \\
\text { a análise. }\end{array}$ & 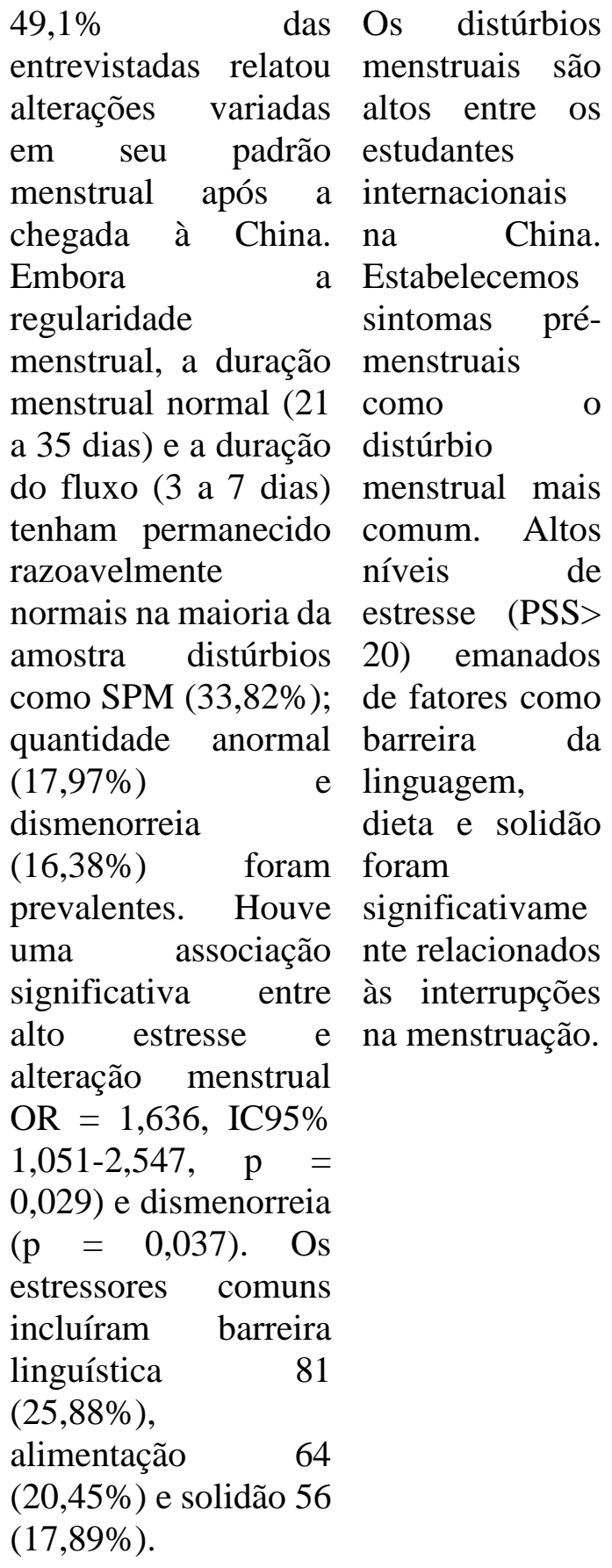 \\
\hline
\end{tabular}

\section{DISCUSSÃO}

Dentre os 14 estudos analisados, a maior prevalência de Síndrome Pré-Menstrual nas populações analisadas foi de $92,3 \%$, a menor, foi de $23,9 \%$ sendo a média de $59 \%$, utilizando os critérios da ACOG, o que indica grande variabilidade de acordo com o contexto da população em análise. Somado a isso, outros estudos relataram a presença de pelo menos um dos sintomas moderados a graves da SPM no ciclo, sendo a maior frequência de $95 \%$, a menor de $72,3 \%$ e a média de todos os dados apresentados $85,4 \%$. Esses dados apresentados demonstram que a maior parte da população em estudo sofre com um agravo dessa doença.

Os sintomas mais apresentados nos estudos foram: depressão $(83,9 \%)$, dor $(83,9 \%)$ e outros sintomas físicos $(64,3 \%)$, estresse $(77,8 \%)$, insônia ou hipersonia (54,6\%), fadiga (59\%) e dismenorreia $(41,9 \%)$. 
Além disso, como impacto negativo no desempenho acadêmico os sintomas da síndrome prémenstrual apresentam prejuízos de autodeterminação e motivação acadêmica, produtividade (48,3\%), atividades de vida social (19\%), relacionamentos $(28,7 \%)$ e na performance em geral $(44,3 \%)$.

Todos esses sintomas, sobretudo os que dizem respeito à produtividade e determinação podem afetar diretamente o desenvolvimento acadêmico de mulheres. Ademais, os outros fatores citados podem afetar no desempenho indiretamente.

Os fatores de risco para a piora dos sintomas da SPM foram bastante variáveis. Um estudo demonstrou que não houve diferença entre os sintomas da síndrome pré-menstrual dependendo da situação sociodemográfica, já outro artigo demonstrou que os sintomas da SPM eram piores em estudantes com uma percepção ruim a muito ruim de sua situação econômica. Foi encontrado um dado significativo que demonstrou que a síndrome pré-menstrual foi mais comum entre estudantes residentes em áreas rurais $(65,1 \%$ de 490 estudantes $)$.

Quanto ao IMC os dados foram controversos, visto que um estudo demonstrou que mulheres com IMC menor que $18,5 \mathrm{~kg} / \mathrm{m}^{2}$ tinham escores de sintomas maiores. Porém, um outro estudo demonstrou que o aumento do IMC tem relação com os sintomas da SPM.

Com relação a alimentação das estudantes encontrou-se que, enquanto a ingestão de alimentos com alto teor calórico, de gordura, açúcar e sal foi associada ao aumento do risco de relatar sintomas físicos (OR 3,2, IC 95\% 1,4- 7,3; p <0,05), o consumo de frutas (OR 0,34, IC 95\% 0,125-0,92; p $<0,05)$ foi associado a uma diminuição do risco de relatar sintomas comportamentais. Os resultados de outro estudo mostraram que existe uma relação significativa entre SPM e consumo de frituras ( $\mathrm{P}$ $=0,017)$, bebidas doces $(\mathrm{P}=0,018)$, fast-food $(\mathrm{P}=0,048)$, frutas $(\mathrm{P}=0,012)$. Foi demonstrado que a síndrome pré-menstrual foi significativamente maior em estudantes que consumiam uma grande quantidade de alimentos gordurosos e com alto teor calórico.

Quanto ao consumo de álcool e tabagismo foi encontrado que a análise de regressão múltipla mostrou que o tabagismo estava associado ao aumento do risco de relatar sintomas psicológicos (OR 2,5, IC 95\% 1,1-5,8; p <0,05) e comportamentais (OR 2,2, IC 95\% 1,0-4,9; p<0,05). Outro estudo mostrou que a SPM foi pior em estudantes que usavam cigarro ( $72,1 \%$ de 490 estudantes) e estudantes com consumo de álcool (65,5\% de 490 estudantes).

Outros fatores de risco que aumentavam os sintomas da SPM foram: mulheres que tinham alguma doença crônica ou anemia $(\mathrm{p}<0,05)$. Outro estudo mostrou também que estudantes com doenças crônicas tinham mais sintomas $(74,1 \%)$. Além disso nenhum exercício habitual $(\mathrm{P}=0,006)$, história familiar de SPM $(\mathrm{P}=0,002)$, circunferência do quadril $(\mathrm{P}=0,04)$ aumentavam os sintomas. Outro dado relevante afirma que níveis mais altos de vitamina D diminui os sintomas de SPM.

\section{CONCLUSÃO}

Após análise da literatura, nota-se que os sintomas de Síndrome Pré-Menstrual interferem na vida das mulheres, afetando em diversos aspectos, sobretudo no meio acadêmico. O reflexo no meio acadêmico é visto pela queda de produtividade, determinação e motivação, além disso, outros sintomas como depressão, dores e sintomas físicos, estresse, insônia e fadiga podem prejudicar indiretamente o desempenho das pacientes. Destaca-se também que o estilo de vida levado pelas estudantes, muitas vezes influenciado pelo contexto em que estão inseridas podem se tornar fatores agravantes para a SPM, lesando ainda mais o desempenho dessas mulheres.

\section{REFERÊNCIAS}

1. Tensão Pré-Menstrual - Critérios para diagnóstico [Internet]. Febrasgo.org.br. 2020 [cited 28 July 2020]. Available from: https://www.febrasgo.org.br/pt/noticias/item/422-tensao-premenstrual-criterios-para-diagnóstico

2. Roriz Mariana Braz Mendes, Lima Camila Melo de Araújo Moura e. A Influência da Tensão Pré-Menstrual nos Sintomas Emocionais e no Consumo Alimentar [Trabalho de Conclusão 
de Curso on the Internet]. [place unknown]: CENTRO UNIVERSITÁRIO DE BRASÍLIA UniCEUB; Faculdade de Ciências da Educação e Saúde; Curso de Nutrição; 2017 [cited 2020 Jul 28]. Available from: https://repositorio.uniceub.br/jspui/bitstream/235/11181/1/TCC\%20Mariana\%20Braz\%20M endes.pdf

3. Takeda T, Imoto Y, Nagasawa H, Muroya M, Shiina M. Premenstrual Syndrome and Premenstrual Dysphoric Disorder in Japanese Collegiate Athletes. Journal of Pediatric and Adolescent Gynecology. [Internet]. 2015 [citado em 27 Jul 2020]. 28(4):215-218. Disponível em: https://www.jpagonline.org/article/S1083-3188(14)00260-5/fulltext

4. Bhuvaneswari K, Rabindran P, Bharadwaj B. Prevalence of premenstrual syndrome and its impact on quality of life among selected college students in Puducherry. Natl Med J India [Internet] 2019 [citado em 27 Jul 2020] 32:17. Disponível em: https://doi.org/10.4103/0970258x.272109

5. Bu L, Lai Y, Deng Y, Xiong C, Li F, Li L et al. Negative Mood Is Associated with Diet and Dietary Antioxidants in University Students During the Menstrual Cycle: A Cross-Sectional Study from Guangzhou, China. Antioxidants [Internet] 2019 [citado em 28 Jul 2020] ;9(1):23. Disponível em: https://www.mdpi.com/2076-3921/9/1/23/htm

6. Fatemi M, Allahdadian M, Bahadorani M. Comparison of serum level of some trace elements and vitamin $\mathrm{D}$ between patients with premenstrual syndrome and normal controls: A crosssectional study. International Journal of Reproductive BioMedicine.[Internet] 2019 [citado em 28 Jul 2020] Disponível em: https://www.ncbi.nlm.nih.gov/pmc/articles/PMC6804325/

7. Shrestha DB, Shrestha S, Dangol D, et al. Premenstrual Syndrome in Students of a Teaching Hospital. J Nepal Health Res Counc.[Internet] 2019. [citado em 27 Jul 2020]. 17(2):253-257. Disponível em: https://doi.org/10.33314/jnhrc.v0i0.1213

8. Victor F, Souza A, Barreiros C, Barros J, Silva F, Ferreira A. Quality of Life among University Students with Premenstrual Syndrome. Revista Brasileira de Ginecologia e Obstetrícia / RBGO Gynecology and Obstetrics. [Internet] 2019 [citado em 27 Jul 2020] 41(05):312-317. Disponível em: https://www.thieme-connect.de/products/ejournals/html/10.1055/s-0039$\underline{1688709}$

9. Hashim MS, Obaideen AA, Jahrami HA, et al. Premenstrual Syndrome Is Associated with Dietary and Lifestyle Behaviors among University Students: A Cross-Sectional Study from Sharjah, UAE. Nutrients. [ Internet] 2019 [citado em 28 Jul 2020] 11(8):1939. Disponível em: https://www.ncbi.nlm.nih.gov/pmc/articles/PMC6723319/

10. Danborno AM, Nwankwo M, Kure J, Eluwa C. Prevalence of Premenstrual Syndrome and Changes in Blood Pressure with Menstrual Cycle Among University Students. Niger J Physiol Sci. [Internet] 2018. [citado em 28 Jul 2020] 33(2):117-124.. Disponível em: https://www.thieme-connect.de/products/ejournals/html/10.1055/s-0039-1688709

11. Mohib A, Zafar A, Najam A, et al.. Premenstrual Syndrome: Existence, Knowledge, and Attitude Among Female University Students in Karachi. Cureus. [Internet] 2018 [citado em 27 Jul 2020] 10(3): e2290. Disponível em: https://www.cureus.com/articles/11142premenstrual-syndrome-existence-knowledge-and-attitude-among-female-universitystudents-in-karachi

12. İşik H, Ergöl Ş, Aynioğlu Ö, Şahbaz A, Kuzu A, Uzun M. Premenstrual syndrome and life quality in Turkish health science students. Turk J Med Sci [Internet] 2016 [citado em 28 Jul 2020] 46: 695-701. Disponível em: https://journals.tubitak.gov.tr/medical/issues/sag-16-463/sag-46-3-16-1504-140.pdf

13. Takeda T, Imoto Y, Nagasawa H, Takeshita A, Shiina M. Fish Consumption and Premenstrual Syndrome and Dysphoric Disorder in Japanese Collegiate Athletes. Journal of Pediatric and Adolescent Gynecology.[Internet] 2016 [citado em 26 Jul 2020] 29(4):386-389. Disponível em: https://www.jpagonline.org/article/S1083-3188(16)00149-2/fulltext

14. Nooh A, Abdul-Hady A, El-Attar N. Nature and Prevalence of Menstrual Disorders among Teenage Female Students at Zagazig University, Zagazig, Egypt. Journal of Pediatric and 
Adolescent Gynecology. [Internet] 2016. [citado em 28 Jul 2020] 29(2):137-142. Disponível em: https://www.jpagonline.org/article/S1083-3188(15)00309-5/fulltext

15. Arslantaş H, Abacigil F, Çinakli Ş. Relationship between premenstrual syndrome and basic personality traits: a cross-sectional study. Sao Paulo Medical Journal. [Internet] 2018 [citado em 28 Jul 2020] ;136(4):339-345. Disponível em: https://www.scielo.br/scielo.php?pid=S1516-31802018000400339\&script=sci_arttext

16. Ansong E, Arhin S, Cai Y, Xu X, Wu X. Menstrual characteristics, disorders and associated risk factors among female international students in Zhejiang Province, China: a crosssectional survey. BMC Women's Health. [Internet] 2019; [citado em 28 Jul 2020] 19(1). Disponível em: https://bmcwomenshealth.biomedcentral.com/articles/10.1186/s12905-0190730-5 